\title{
Biossegurança e gerenciamento de resíduos de serviços de saúde: a importância na formação do profissional da Odontologia na perspectiva da saúde humana e ambiental
}

\author{
Carmen Angela Guimarães Leal*
}

\footnotetext{
* Professora e coordenadora de biossegurança da Faculdade de Odontologia de Valença.
}

\section{RESUMO}

A construção da consciência de cidadania, com responsabilidade e ética, depende de ações que iniciem na formação e possam ser perpetuadas no trabalho profissional. É no período da graduação da odontologia que o aluno se vê à frente com questões não somente técnicas, mas com as situações da saúde na visão da integralidade, como no caso dos resíduos gerados durante a prática clínica. Os agravos aos profissionais/alunos e funcionários, a terceiros nos ambientes extraestabelecimento, e também ao meio ambiente poderão ocorrer se procedimentos corretos no gerenciamento de resíduos produzidos na odontologia não forem observados, pois os potenciais de risco destes aumentam quando os mesmos são manuseados de forma inadequada ou não são apropriadamente acondicionados e descartados, especialmente em situações que favoreçam a penetração de agentes de risco no organismo e/ou agridam o meio ambiente. Na problemática que envolve estes resíduos, o conhecimento é a ferramenta essencial para ações de manejo adequado, desde a minimização na produção até a disposição correta. Com o intuito de contribuir como o conhecimento pode ser inserido na aplicação desta temática na formação do profissional da odontologia, propõe-se uma revisão da literatura, com pesquisa descritiva, exploratória e bibliográfica de materiais acadêmico-científicos e normativos relacionados ao gerenciamento de resíduos de serviços de saúde na odontologia, associando à importância da abordagem deste tema na disciplina de Biossegurança, apresentando o seu escopo de promotora em saúde humana e ambiental, que se configura como relevante proposta no âmbito da saúde pública.

Descritores: Educação em odontologia. Biossegurança.Resíduos de Serviços de Saúde.

\section{INTRODUÇÃO}

A problemática que envolve os resíduos desafia a sociedade moderna e é um tema que compreende diversos setores, como indústria, trabalho, meio ambiente, saúde e educação, e necessita de políticas públicas na organização de um sistema de gestão que se relaciona diretamente com a saúde pública ${ }^{1}$. Os resíduos de serviços de saúde (RSS) fazem parte dos resíduos sólidos urbanos e são aqueles gerados em serviços relacionados com o atendimento à saúde humana ou animal. São classificados 
conforme a Resolução 358/2005 do Conselho Nacional do Meio Ambiente (CONAMA), divididos em grupo A, que são os resíduos com a possível presença de agentes biológicos; grupo $\mathrm{B}$, que agrega os resíduos contendo substâncias químicas que podem apresentar risco à saúde pública ou ao meio ambiente; grupo $\mathrm{C}$, que são quaisquer materiais resultantes de atividades humanas que contenham radionuclídeos; grupo D são resíduos que não apresentam risco biológico, químico ou radiológico à saúde ou ao meio ambiente, podendo ser equiparados aos resíduos domiciliares; grupo $\mathrm{E}$, que são os materiais perfurocortantes ou escarificantes ${ }^{2}$.

O desenvolvimento tecnológico, a complexidade da atenção na área da saúde e o aumento do número de instituições de saúde propiciaram um acréscimo na geração de produtos neste setor de prestação de serviços, principalmente de materiais descartáveis. As informações dos riscos relacionados a estes resíduos impuseram um gerenciamento diferenciado e propiciaram pesquisas com o intuito de trazerem soluções quanto ao tratamento e disposição final destes, ao mesmo tempo que auxiliou no conhecimento do manejo, ao criar as normas legisladoras ${ }^{1}$.

A Odontologia, como profissão da área da saúde, também gera RSS dos grupos A, B, D e E em seus ambientes de serviço que, de forma geral, não produzem quantidades consideráveis destes resíduos quanto os grandes geradores, como as instituições hospitalares, por exemplo. Contudo, o contingente em número cada vez maior de profissionais no mercado de trabalho gera uma fração considerável de resíduos que, se não tiverem o gerenciamento adequado por parte dos geradores, ocasionam diversos problemas relacionados aos seus riscos ${ }^{1}$.

A Biossegurança, conteúdo que abrange a prevenção de riscos à saúde humana e ambiental é ampla em sua concepção e envolve não só a prevenção de doenças infectocontagiosas na relação profissional-paciente no meio ambiente ocupacional, mas também as questões que abrangem a saúde do trabalhador que lida diretamente na coleta e transporte dos resíduos de origem odontológica, bem como àqueles que subsistem dos resíduos. $\mathrm{E}$, quando medidas preventivas adequadas não são devidamente aplicadas quando da destinação final dos resíduos, a contaminação do meio ambiente pode ocorrer, comprometendo a saúde da população local ${ }^{1}$.

Os princípios de Biossegurança no emprego de medidas técnicas, administrativas e normativas para prevenir acidentes, preservando a saúde pública e o meio ambiente são considerados pela Agência Nacional de Vigilância Sanitária (ANVISA, 2004), ao instituir a Resolução 306, que dispõe sobre o Regulamento Técnico para o gerenciamento de resíduos de saúde ${ }^{3}$. O gerenciamento de resíduos de serviços de saúde da prática odontológica deve estar relacionado às normas de Biossegurança para a preservação e proteção da saúde dos trabalhadores, da comunidade e meio ambiente e que, segundo a Organização Mundial de Saúde (OMS, 2007), a gestão segura e sustentável de RSS é um imperativo de saúde pública e uma responsabilidade de todos, pois o manejo inadequado destes resíduos apresenta um risco significativo para os pacientes, profissionais de saúde, a comunidade e o meio ambiente ${ }^{4}$. 
$\mathrm{Na}$ odontologia, a disciplina de Biossegurança tem um papel primordial, pois nos cursos de formação objetiva prover ao futuro profissional o conhecimento de todos os riscos que estão presentes em seu ambiente de trabalho e nos processos que desenvolvem, com vistas à proteção da saúde do profissional, auxiliar, paciente e terceiros, bem como do meio ambiente, no caso do manejo de resíduos. Esta disciplina tem uma atuação interdisciplinar, fortalecendo os saberes relacionados a outras áreas do conhecimento, a fim de proporcionar um entendimento mais amplo ao educando, estimulando-o, no seu papel dentro da sociedade, à responsabilidade, à qualidade e à ética no seu fazer profissional ${ }^{1}$.

$$
\text { Considera-se importante }
$$
compreender como o gerenciamento de resíduos possibilita que processos educacionais possam interferir, de forma positiva e necessária, norteando para desenvolvimento de competências, estimulando a capacidade de construção de cidadãos com responsabilidades, além da promoção da saúde bucal, mas também visando a preservação e promoção da saúde humana, através da saúde ambiental e ocupacional.

Nessa perspectiva, a partir da revisão da literatura através de pesquisa descritiva, exploratória e bibliográfica, utilizando teses, dissertações, artigos, normas e legislações, o artigo propõe, ao apresentar a Biossegurança e sua relação com os riscos nas práticas da odontologia, descrever o gerenciamento de resíduos de serviços da odontologia como um campo do conhecimento pertinente à área, aplicado como conteúdo importante para o graduando, objetivando orientar na formação responsável e ética do futuro profissional quanto à saúde humana e ambiental, com vistas à saúde pública.

\section{REVISÃO DA LITERATURA}

\section{A Biossegurança e os riscos na odontologia}

A concepção mais ampla de Biossegurança a traduz como um conjunto de ações que se destinam à segurança da vida $^{5}$. Em termos da construção do conhecimento da Biossegurança, visando sua aplicabilidade universal, sua proposta conceitual a definiu como:

"O conjunto de saberes direcionados para ações de prevenção, minimização ou eliminação de riscos inerentes às atividades de pesquisa, produção, ensino, desenvolvimento tecnológico e prestação de serviços, as quais possam comprometer a saúde do homem, dos animais, das plantas e do meio ambiente ou a qualidade dos trabalhos desenvolvidos." 6

Segundo Costa e Costa ${ }^{5}$ (2009), sua aplicação prática e cognitiva pode se expressar em várias abordagens como módulo, processo ou conduta. Como módulo, pode assumir um valor interdisciplinar através das matrizes curriculares dos seus cursos e programas; como processo, pela ação educativa, formal e prática, podendo ser representada por um sistema ensino-aprendizagem, em um processo de aquisição de conteúdos e habilidades a serem aplicados nas atividades práticas onde a Biossegurança faz-se obrigatória, uma vez que seu objetivo original é preservar a saúde do homem e do meio ambiente; conduta, na análise do somatório de conhecimentos, hábitos, comportamentos e sentimentos, devendo ser incorporados ao homem, para 
desenvolver, de forma segura, sua atividade profissional.

Os textos e manuais relacionados à Biossegurança, editados em todo o mundo, apontam-na como um conceito mais amplo, em que o risco - ou a probabilidade de determinado dano ocorrer, passa a ser objeto da pesquisa dessa nova ciência, sendo que o risco biológico, ao qual estão sujeitos os pesquisadores ou profissionais que atuam em laboratórios e ambientes nos quais estão presentes micro-organismos, é apenas um dos segmentos de atuação da Biossegurança, como disciplina científica ${ }^{7}$.

A prevenção de riscos à saúde ambiental e humana é objetivo central da Biossegurança, a qual dialoga e se apropria de saberes imprescindíveis de outras áreas do conhecimento científico, o que caracteriza a interdisciplinaridade do campo $^{8}$. Com isso, todas estas definições mostram que a Biossegurança envolve relações entre o homem, a sociedade, a biodiversidade, os agentes biológicos, a tecnologia e o risco 9 .

Para todos os processos que envolvem a área profissional da saúde, a Biossegurança é um importante instrumento de controle que favorece a elaboração dos parâmetros de qualidade, que se reflete na saúde do trabalhador, do ambiente, dos produtos e dos serviços. A observância dos procedimentos estimula a dinâmica de conhecimentos e informações, gerando melhores processos, serviços, equipamentos e materiais para a prevenção dos riscos nos ambientes ocupacionais ${ }^{1}$.

A odontologia é um campo da saúde onde o profissional convive com riscos potenciais e manifestos no cotidiano de suas atividades práticas, no qual a classificação dos riscos, segundo a Norma Regulamentadora (NR) 09 Programa de Prevenção de Riscos Ambientais, da Portaria 3.214, do Ministério do Trabalho e Emprego, de 1978 (alterada e atualizada através da Portaria $\mathrm{n}^{\mathrm{o}} 25$ de 29 de dezembro de 1994) ${ }^{10}$, podem ser listados como:

Riscos biológicos: objetos perfurocortantes, respingos e aerossóis contendo matéria orgânica (sangue, saliva, exsudato purulento, restos de tecidos etc) que podem ser fontes de infecções virais, como hepatites $\mathrm{B}$ e $\mathrm{C}$, gripe, herpes e AIDS, e de infecções bacterianas, como a sífilis e tuberculose, além de contaminações por outros patógenos, como fungos e príons.

Riscos químicos: a exposição ao vapor de mercúrio das restaurações de amálgama; gases anestésicos (gás óxido nitroso); lâminas de chumbo das películas radiográficas; desinfetantes, saneantes, medicamentos (vernizes, formocresol, cimentos, entre outros), monômeros e polímeros do acrílico, materiais de moldagem, entre outros; reações alérgicas (luvas de látex, solventes, polímeros do acrílico, óleos lubrificantes, detergentes, efluentes radiográficos, entre outros).

Riscos físicos: radiação ionizante (raios$\mathrm{X}$ ), radiação não ionizante (luz fotopolimerizável e laser), ruídos, temperatura.

Riscos de acidentes: iluminação, instrumentos de cortes, abrasão e perfurantes.

Riscos ergonômicos: problemas músculoesqueléticos (relação direta com posições posturais inadequadas e movimentos repetitivos prolongados) e problemas circulatórios (varizes, hemorróidas); psicossociais (estresse devido ao 
ritmo e intensidade das tarefas, metas de produtividade, insatisfação social e pessoal, entre outros).

De acordo com Leal ${ }^{1}$ (2012), apesar da Biossegurança ser um campo do conhecimento relacionado, de forma geral, aos riscos biológicos, sua proposta deve ser compreendida para além dos limites envolvendo os procedimentos relacionados ao controle de infecção e contaminação. A prevenção destes riscos tem um papel muito importante na Biossegurança, através dos diversos processos, como métodos de esterilização, desinfecção e assepsias, uso de equipamentos de proteção individual (EPI), imunizações da equipe odontológica, precauções de acidentes com material biológico, entre muitos outros, para o controle de infecção cruzada. Embora o risco biológico seja o mais observado pelos profissionais atuantes no setor da saúde, outros tipos de risco compõem o escopo de reflexão e aplicação da Biossegurança ${ }^{1}$.

Devido, portanto, aos qualitativos e quantitativos de riscos associados à profissão, a odontologia necessita do suporte da Biossegurança como orientadora de procedimentos de atuação permanente em todos os seus níveis, que atua na formação destes profissionais, disponibilizando conhecimentos relacionados aos variados riscos decorrentes do ambiente odontológico a que todos os envolvidos estão expostos: profissional, auxiliar, paciente e técnico de prótese, bem como terceiros e o meio ambiente, quando relacionado ao manejo dos resíduos ${ }^{1}$.

\section{Os resíduos de serviços de saúde da prática odontológica}

A prática odontológica produz variados tipos de resíduos devido a diversidade de materiais utilizados, bem como as especificidades de suas clínicas. Todas as clínicas odontológicas geram resíduos químicos (grupo B) como saneantes e desinfetantes, necessários para a limpeza e desinfecção do ambiente, equipamentos e artigos, bem como resíduos do grupo $\mathrm{D}^{1}$.

De forma geral, as clínicas e consultórios odontológicos possuem aparelhos de raios $\mathrm{X}$ para realizarem os exames radiográficos em seus pacientes, gerando resíduos químicos, como reveladores e fixadores, filmes radiográficos e lâminas de chumbo; algumas especialidades odontológicas também geram resíduos químicos específicos, como restos de amálgamas, materiais de moldagens, cimentos, vernizes, polímeros e monômeros, medicamentos antimicrobianos, citando apenas alguns. E nas clínicas, os resíduos dos grupos A (órgãos dentários e materiais com restos de tecidos, sangue, saliva e exsudato purulento) e E (materiais perfurocortantes e escarificantes contaminados ou não com microrganismos) são diversificados, principalmente em quantidade ${ }^{1}$.

Para que ocorra o manejo correto, os resíduos devem ser classificados de acordo com a Resolução CONAMA 358/2005², bem como a RDC 306/2004 da ANVISA $^{3}$. Este regulamento da ANVISA descreve:

"O gerenciamento dos resíduos de serviços de saúde constitui-se em um conjunto de procedimentos de gestão, planejados e implementados a partir de bases científicas e técnicas, normativas e legais, com o 

profissional da Odontologia na perspectiva da saúde humana e ambiental

objetivo de minimizar a produção de resíduos e proporcionar aos resíduos gerados um encaminhamento seguro, de forma eficiente, visando à proteção dos trabalhadores, a preservação da saúde pública, dos recursos naturais $e$, organizando para este fim, o Regulamento Técnico para o Gerenciamento dos Resíduos de Serviços de Saúde. "3

Todo gerador deve elaborar um Plano de Gerenciamento de Resíduos de Serviços de Saúde (PGRSS), baseado nas características dos resíduos gerados e na sua classificação. Este documento deve ser compatível com as normas locais relativas à coleta, transporte e disposição final dos resíduos gerados nos serviços de saúde, normas estas estabelecidas pelos órgãos locais responsáveis por estas etapas $^{3}$.

A Agenda 21, como documentobase de que cada país dispõe para construir o seu plano de preservação do meio ambiente, elaborada a partir da Conferência das Nações Unidas sobre o Meio Ambiente e o Desenvolvimento, conhecida como "Rio-92" e "Eco-92", orienta na Seção II - "Conservação e gestão dos recursos para o desenvolvimento" ${ }^{11}$ :

"Os itens distribuídos e relacionados à gestão dos resíduos e suas disposições, que possuem a finalidade de buscar soluções integradas e compatíveis básicas de minimização de resíduos, reciclagem e reutilização, tratamento $e$ disposição ambientalmente seguros, substituição de matérias-primas perigosas, transferência e desenvolvimento de tecnologias limpas, devendo nortear, em âmbito mundial, as ações dos governantes, organizações e grupos setoriais responsáveis pela gestão de resíduos.

A classe odontológica, como organização e/ou grupo setorial, dispõe das normas e legislações já citadas e pertinentes ao âmbito dos serviços de saúde, para auxiliar os profissionais na empreitada do gerenciamento de resíduos. O conhecimento destas regulamentações orienta-nos em compromissos frente às responsabilidades dos riscos dos resíduos gerados em seus ambientes laborais ${ }^{1}$.

\section{A disciplina de Biossegurança e o gerenciamento de resíduos na formação do profissional de odontologia}

As Diretrizes Curriculares dos Cursos de Graduação da área de Saúde, em particular da odontologia, elaboradas pelas Comissões de Especialistas de Ensino e encaminhadas pela SESu/MEC ao Conselho Nacional de Educação e publicadas em 2002 12 , apontam para algumas competências e habilidades que devem contemplar o perfil do profissional da saúde. Apesar da formação necessária para capacitar o futuro profissional preparado para o mercado de trabalho que o espera, faz-se necessário que o egresso seja um profissional capacitado também nos aspectos éticos/bioéticos, humanistas e de cidadania e não apenas nos aspectos técnicos, característicos do modelo biomédico $^{13}$.

Com as necessidades prementes e atuais das profissões da área da saúde, a formação acadêmica, em conformidade com as diretrizes curriculares, direciona o aluno para uma postura profissional mais integral, com base nos princípios do 
Sistema Único de Saúde (SUS). Nesse contexto, uma visão mais ampla também deve ser estimulada, no qual novas formas de organizações curriculares mais integradoras das disciplinas orientam para métodos de ensino que favoreçam um processo de construção de conhecimentos mais significativos à formação humana $\mathrm{e}$ profissional do aluno ${ }^{1}$.

Estas condições permitem que o cirurgião-dentista seja formado para se tornar não somente um profissional habilitado, capaz de prevenir, tratar e manter a saúde bucal, mas antes de tudo ser um promotor de saúde, sensibilizado para uma prática odontológica interdisciplinar no âmbito coletivo. Isso pressupõe a formação de um profissional que domine uma gama de conhecimentos e habilidades técnicas das áreas de saúde e administração, com visão ampliada do contexto em que está inserido, além de um forte compromisso social ${ }^{13}$.

Sêcco et al. ${ }^{14}$ (2003) orientam que atenção especial deva ser dada aos currículos escolares na formação dos profissionais de saúde, embasando seus alunos para que possam pensar a realidade dos trabalhadores e atuar de maneira compatível com a promoção da saúde. No ensejo apresentado pelos autores, inclui-se os conhecimentos sobre o gerenciamento de resíduos de serviços de saúde, tema importante e atual que deve ser explorado na rotina das relações de trabalho na área da saúde.

$\mathrm{Na}$ abordagem de RSS, o conhecimento pertinente pode significar um caminho de extrema relevância, tanto para a saúde humana quanto para a ambiental, pois sabe-se que não é possível prevenir e proteger a saúde individual e coletiva sem cuidar igualmente do meio ambiente, uma vez que qualquer dano e desequilíbrio a esse meio acaba por repercutir sobre a saúde humana, trazendo-lhe agravos ${ }^{15}$.

A educação formal continua se constituindo num espaço importante para o desenvolvimento de valores e atitudes comprometidas com a sustentabilidade ecológica e social. Para estabelecer conexões entre teoria e prática, as escolas representam espaços privilegiados, com possibilidades de estimular os alunos a desenvolver uma postura de cidadãos, conscientes das suas ações e percebendose como parte integrante do meio ambiente $^{16}$.

É necessário que o ensino seja embasado em uma prática emancipatória e transformadora para a abordagem de RSS nos cursos de graduação da área da saúde, para que este saber não seja apenas um conhecimento de como praticar, mas o ambiente de formação propicie reflexão, problematização, crítica, articulação e comprometimento com a construção de sujeitos que incorporem posturas éticas, de solidariedade, de consciência cidadã, de compromisso social, atuando de forma responsável para com o meio ${ }^{17}$.

Ao não ser dada a devida importância aos conhecimentos e principalmente ao manejo adequado no que se refere aos resíduos gerados nestes ambientes, observam-se não concordância às normas e legislações, embasadas em estudos e trabalhos científicos quanto aos procedimentos corretos e de menor ou nenhum impacto à saúde humana $\mathrm{e}$ ambiental. E as não conformidades no gerenciamento de resíduos pelos cirurgiões-dentistas, discutidas em diversos trabalhos de pesquisas na literatura científica, apresentam uma 
lacuna que sugere uma provável não inclusão de conhecimentos sobre os resíduos de modo incisivo na formação acadêmica do profissional ${ }^{1}$.

Participando dessa reflexão, Corrêa et $a l .{ }^{17}$ (2005) observam que é possível que a não inserção de abordagem de RSS no processo de formação dos futuros profissionais da saúde seja um aspecto importante para justificar o que acontece hoje em relação a esses resíduos, tanto nos estabelecimentos de saúde, como no meio ambiente. E é premente a necessidade de investir na formação dos profissionais da área de saúde, a fim de alcançar as mudanças de condutas consideradas ocupacional e ambientalmente nocivas ${ }^{18}$.

Ao não existir programas de treinamento dentro da instituição para os profissionais - sendo que até mesmo professores desconhecem esse assunto, acarreta, consequentemente, na não transmissão dessas informações para os alunos, que por sua vez saem da universidade sem a devida formação quanto aos $\mathrm{RSS}^{19}$.

Esta afirmativa é corroborada por Moreschi $^{15}$ (2013) no seu trabalho de pesquisa com docentes, alunos e egressos de cursos formadores da área da saúde, ao apresentar que os egressos expõem que, de modo geral, durante a formação acadêmica a temática dos RSS foi abordada superficialmente, tanto nas aulas práticas quanto teóricas; os alunos apontam lacunas por parte da instituição formadora, pois acreditam que a temática deva ser melhor discutida; e os docentes veem a necessidade da questão dos resíduos de serviços de saúde ser abordada numa disciplina exclusiva, e que por meio dessa estratégia torna-se possível contemplar saberes acerca dos RSS em todos os cursos da área da saúde.

A necessidade de enfatizar essa questão no processo de formação requer considerar que os indivíduos que atuam nestes espaços tenham a construção de uma consciência ética, em busca da qualidade de vida e desenvolvimento sustentável $^{20}$. E sem o ensino nas universidades, formam-se profissionais que possivelmente não realizarão um manejo adequado dos RSS em seus consultórios e clínicas particulares, já que não conhecem a legislação vigente ${ }^{19}$.

Ampliando para o conhecimento pertinente à Biossegurança, aos riscos e à saúde ocupacional e ambiental, o aluno e o trabalhador não pode ser um mero reprodutor, mas sim um agente participativo-transformador no seu ambiente ocupacional, conjugando o saber "fazer-ser-aprender". O processo educativo envolve uma ação de reflexão, e deve ultrapassar a ideia da simples normatização e abranger, inclusive, aspectos relativos à ética, já que esta está implícita em praticamente todas as ações que envolvem a Biossegurança ${ }^{21}$.

As medidas tomadas no contexto da Biossegurança, aliadas à economia de recursos, preservação do meio ambiente, ética e responsabilidade certamente irão garantir maior qualidade de vida para os profissionais de saúde, além de manter a segurança do ambiente de trabalho $\mathrm{e}$ também externamente a este. $\mathrm{O}$ ensino contextualizado da Biossegurança, relacionado aos saberes prévios dos alunos e do cotidiano, pode ser uma estratégia eficaz para o desenvolvimento de competências nesse campo, que é cada vez mais exigido no mundo globalizado 
atual, principalmente nas relações trabalho-saúde ${ }^{21}$.

Costa e $\operatorname{Costa}^{9}$ (2010) ressaltam que a fraca participação do sistema formal de ensino, no que se refere à Biossegurança, ocasiona dificuldades que se refletem nas suas ações ocupacionais, abrindo com isso, espaços para a ocorrência de agravos à saúde a ao meio ambiente. E a prevenção de riscos à saúde ambiental e humana é objetivo central da Biossegurança, a qual dialoga e se apropria de saberes imprescindíveis de outras áreas do conhecimento científico, o que caracteriza a interdisciplinaridade do campo $^{8}$. Desse modo, a disciplina de Biossegurança possibilita a interdisciplinaridade entre as variadas disciplinas e campos do saber, no qual a promoção da saúde seja a base sustentável de sua proposta pedagógica ${ }^{1}$.

A construção participativa e crítica entre as diferentes ciências, proposta pela Biossegurança, permite que problemas atuais e inerentes a todos, como resíduos e questões ambientais, possam estimular a discussão entre os envolvidos no processo ensino-aprendizagem, trazendo a importância destas questões para dentro do ambiente de estudo, apresentando a responsabilidade que cabe a cada um na proposta de promover a saúde humana $\mathrm{e}$ ambiental $^{15}$.

Nessa perspectiva, por meio da interdisciplinaridade, é possível favorecer a ampliação do conhecimento sobre RSS na ótica do despertar de uma consciência socioambiental, subsidiando o saber agir profissional nas questões que envolvem a produção destes resíduos. Desta forma, é importante a construção de uma pedagogia ambiental e transformadora, sendo que essa pedagogia deve englobar as múltiplas esferas da vida planetária e social, inclusive a individual, ou caso contrário, o processo educativo não pode ser considerado transformador ${ }^{15}$.

E a educação ambiental, enquanto práxis social, coopera para o processo de construção de uma sociedade embasada por novos padrões civilizacionais e societários diferentes dos atuais, no qual a sustentabilidade da vida, a atuação política consciente e a construção de uma ética ecológica seja sua essência. Revela-se, com isso, necessária a formação de profissionais qualificados e com conhecimento da importância de manuseio dos RSS, para que se possa educar/formar com vistas à sustentabilidade ambiental ${ }^{22}$.

A interdisciplinaridade tem sido considerada importante estratégia para dar conta da complexidade que caracteriza o mundo e seus desafios. Dentre eles, os problemas de saúde, que são considerados um cenário eminentemente interdisciplinar, e a integração de disciplinas no âmbito de cursos que preparam recursos humanos para atuar nesse campo poderão certamente levar à formação de profissionais mais comprometidos com a realidade de saúde e com a sua transformação ${ }^{23}$.

As instituições de ensino superior (IES), enquanto formadoras, devem inserir a discussão da Biossegurança como forma de garantir comportamentos preventivos pelo futuro profissional, no resgate da responsabilidade de sua saúde e de terceiros. Um momento único e oportuno deve ser criado, como maneira sistematizada de promover o aprendizado em Biossegurança, sendo que a inserção de temas, como o gerenciamento de RSS, tenha a garantia na formação acadêmica responsável e crítica, e sejam necessárias 

profissional da Odontologia na perspectiva da saúde humana e ambiental

como meio para uma graduação de excelência. Com isso, os processos educacionais de Biossegurança devem ser pedagogicamente estruturados para que sejam capazes de gerar as competências atualmente demandadas para os profissionais da saúde em todos os níveis ${ }^{15}$.

As IES precisam dar o exemplo aos seus graduandos, tendo em vista que cada profissional/graduando irá realizar suas atividades práticas munido de conhecimento e responsabilidade quanto aos riscos decorrentes do descarte inadequado dos RSS. E como estas instituições formarão futuros gestores e profissionais de saúde, no campo acadêmico faz-se necessário potencializar o envolvimento e a participação de toda a comunidade universitária na construção e implantação de políticas para uma gestão adequada dos seus resíduos, para que se possa contribuir de forma efetiva e comprometida com a sustentabilidade ambiental $^{15}$.

A educação continuada também deve fazer parte das estratégias para incentivar o conhecimento quanto ao gerenciamento correto dos resíduos gerados nestes ambientes de serviços de saúde, porém necessário se faz reavaliar como este material poderia ser incluído como conteúdo a ser explorado nos cursos de pós-graduações, bem como periódicos, boletins e jornais impressos e meio eletrônico, entre outros, auxiliando na informação do profissional, pois são temas inerentes à profissão, ou seja, todos produzem resíduos, de forma e quantidade diversas ${ }^{1}$.

Moreschi $^{15}$ (2013) ressalta ser importante o conhecimento ofertado durante a formação acadêmica, porém também é relevante o comprometimento por parte dos gestores na esfera governamental e das instituições de saúde, sendo que há necessidade de consciência por parte dos gestores para implantar políticas públicas voltadas para os resíduos de serviços de saúde, visto que é importante compartilhar responsabilidades entre governo e sociedade. Leal ${ }^{1}$ (2012) corrobora esta observação, afirmando que para o gerenciamento dentro e fora do estabelecimento seja eficaz é necessário que o poder público se envolva e estabeleça leis e regulamentos sobre a gestão de RSS, assumindo o seu papel de gestor local.

Uma aprendizagem significativa deve estar engajada no desenvolvimento de políticas públicas saudáveis e estimular a criação de ambientes favorecedores da saúde, da mudança de comportamentos que permitam a proteção do meio ambiente, a conservação de recursos naturais e o envolvimento cada vez maior da população em projetos de promoção da saúde ${ }^{15}$.

Para que haja o desenvolvimento sustentável, a preservação ambiental e as condições de saúde favoráveis são questões essenciais, pois situações de risco provocadas pela degradação ambiental e/ou por condições de saúde precárias comprometem os pilares da sustentabilidade ${ }^{8}$.

\section{CONSIDERAÇÕES FINAIS}

A Biossegurança e suas recomendações, das mais gerais às mais específicas, devem compor o processo de formação dos profissionais da saúde. $\mathrm{Na}$ odontologia, a valorização da Biossegurança incluída na formação acadêmica oferecida pelas instituições de ensino tem oportunidade de problematizar 
questões relacionadas ao risco ambiental e humano (incluindo o ocupacional) no processo de geração de resíduos na prática odontológica, propondo reflexões a partir da rotina clínica.

Ao estimular nos alunos a percepção de risco orientada pela Biossegurança, elabora-se assim um importante processo cognitivo que associa vários aspectos do risco ligado ao exercício da prática odontológica, onde se destaca o potencial de risco dos resíduos e a problematização da responsabilidade profissional e ética, favorecendo a consolidação e a difusão de uma consciência preventiva voltada para a saúde ocupacional e para saúde ambiental.

Para os futuros profissionais de odontologia, tanto para o desempenho das atividades práticas quanto para a construção de novas percepções que estimulam reflexões que enriquecem a dinâmica acadêmica, é fundamental a compreensão clara da relação entre risco, saúde ocupacional, saúde ambiental, Biossegurança e gerenciamento de resíduos.

Nessa conjuntura, apresenta-se a abertura de um espaço de formulação reflexiva, que passa a compor o campo da Biossegurança, o qual assume a função mediadora de harmonizar as apreensões da sociedade e as questões afetas aos campos da saúde, do ambiente e da ética com as demandas científico-tecnológicas, na busca de um modelo de desenvolvimento sustentável.

$$
\text { As variadas instituições }
$$

formadoras do curso de odontologia devem objetivar integrar aos conhecimentos dos acadêmicos os aspectos relacionados aos resíduos e à educação ambiental, para futuramente, na prática profissional, estes possam ter uma postura mais ética e consciente do seu papel, como profissionais de saúde, não somente oral, mas com a saúde integral.

\section{REFERÊNCIAS}

1. Leal CAG. Estudo da percepção do profissional quanto a Biossegurança no gerenciamento de resíduos odontológicos no município de Valença-RJ. Rio de Janeiro. Dissertação [Mestrado] - Fundação Oswaldo Cruz, Instituto de Pesquisa Clínica Evandro Chagas; 2012.

2. Brasil. Ministério do Meio Ambiente. Conselho Nacional do Meio Ambiente. Resolução no 358, de 29 de abril de 2005. Dispõe sobre o tratamento e disposição final dos resíduos de serviço de saúde e dá outras providências. Diário Oficial da União abr 2005; Seção1.

3. Brasil. Ministério da Saúde. Agência Nacional de Vigilância Sanitária. Resolução RDC n ${ }^{\circ}$ 306, de 07 de dezembro de 2004. Dispõe sobre o Regulamento Técnico para o gerenciamento de resíduos de serviço de saúde. Diário Oficial da União dez 2004; Seção 1.

4. Organização Mundial da Saúde. Environmental health update. Sustainable Development and Healthy Environments. 2007; 2(5) [acesso em: 12 mai 2010]. Disponível em: http://www.searo.who.int/link files/ sde_eh-vol2-no-5.pdf.

5. Costa MAF, Costa MFB. Biossegurança de A a Z. 2ed. Rio de Janeiro: Publit; 2009.

6. FIOCRUZ. Manual de Biossegurança. 2005 [acesso em: 12 de nov 2011]. Disponível em: 

profissional da Odontologia na perspectiva da saúde humana e ambiental

http://www.fiocruz.br/biosseguranca/

Bis/bismanua.htm

7. Oda LM, Santos BC. Memórias da biossegurança e biosseguridade: de Asilomar à biologia sintética. In: Manual de Biossegurança. Hirata MH, Hirata RDC, Mancini Filho J. 2 ed. Barueri, SP: Manole; 2012.

8. Rocha SS, Bessa TCB, Almeida AMP. Biossegurança, proteção ambiental e saúde: compondo o mosaico. Ciênc Saúde Col. 2012; 17(2): 287-292.

9. Costa MAF, Costa MFB. Entendendo a biossegurança: epistemologia e competências para a área de saúde. 2ed. Rio de Janeiro: Publit; 2010.

10. Brasil. Ministério do Trabalho e Emprego. NR 9 - Programa de Prevenção de Riscos Ambientais: Publicada em Portaria GM n. ${ }^{\circ}$ 3.214, de 08 de junho de 1978. Diário Oficial da União jun 1978; Seção 1.

11. Brasil. Ministério do Meio Ambiente. Agenda 21 [acesso em: $12 \mathrm{dez} 2012$ ]. Disponível em: http://www.ana.gov. br/AcoesAdministrativas/RelatorioGe stao/Agenda21/iniciar.html

12. Brasil. Ministério da Educação e do Desporto. Conselho Nacional de Educação. Câmara de Educação Superior. Resolução CNE-CES 3, de 19/02/2002 [acesso em: 20 set 2013]. Disponível em: http://portal.mec.gov. br/cne/arquivos/pdf/CES032002.pdf.

13. Costa ICC. Os sete saberes necessários à educação do futuro e o planejamento das ações de saúde: algumas reflexões e confluências. Rev ABENO. 2007; 7(2):122-9.

14. Secco IAO, Robazzi MLCC, Gutierrez PR, Matsuo T. Acidentes de trabalho e riscos ocupacionais no diaa-dia do trabalhador hospitalar: desafio para a saúde do trabalhador. Rev Espaço Saúde. 2003; 4(1) Disponível em: http://www.uel.br/ ccs/espacoparasaude/v4n1/doc/hospit al.htm.

15. Moreschi C. Resíduos de serviços de saúde: percepção de docentes, discentes e egressos da área da saúde de duas instituições comunitárias de ensino superior do RS. Lajeado. Dissertação [Mestrado] - Centro Universitário Univates, Programa de Pós-Graduação em Ambiente e Desenvolvimento; 2013.

16. Lima W. Aprendizagem e classificação social: um desafio aos conceitos. Fórum Crítico da Educação. Revista do ISEP. 2004; 3(1):29-56.

17. Corrêa LB, Lunardi VL, De Conto SM, Galiazzi MC. O saber resíduos sólidos de serviços de saúde na formação acadêmica: uma contribuição da educação ambiental. Interface Comun Saúde Educ. 2005; 9(18):571-84.

18. Costa EMP. Destinação final dos resíduos sólidos de serviço de saúde da Cidade do Crato - CE. Crato. Monografia [Especialização] Universidade Regional do Cariri, Centro de Ciências e Tecnologia; 2004.

19. Fornaciari KV. Avaliação das práticas de manejo de resíduos de serviços de saúde (RSS) na Faculdade de Odontologia/UERJ. Rio de Janeiro. Dissertação [Mestrado] Universidade do Estado do Rio de Janeiro, Curso de Engenharia Ambiental; 2008.

20. Fernandes MM. Conhecimento dos formandos em Odontologia sobre o 
plano de gerenciamento de resíduos de serviços de saúde: aspectos éticos e legais. Campinas. Monografia [Especialização] - Universidade Estadual de Campinas, Faculdade de Odontologia de Piracicaba; 2009.

21. Pereira MEC, Silva PCT, Costa MAF, Jurberg C, Borba CM. A importância da abordagem contextual no ensino de biossegurança. Ciênc Saúde Col. 2012; 17(6):1643-8.

22. Loureiro CFB, Layrargues PP, Castro RS. (Orgs.). Educação Ambiental: repensando o espaço da cidadania. São Paulo: Cortez, 2002.

23. Vilela EM, Mendes IJM. Interdisciplinaridade e saúde: estudo bibliográfico. Rev Latino-Am Enfermagem. 2003;11(4):525-31.

\section{ABSTRACT \\ Biosafety and waste management in health services: the importance in the dentistry professionals' formation in perspective of human and environmental health}

The building of consciousness in citizenship, with responsibility and ethics, depends on actions that initiate in the formation and can be perpetuated in professional work. It's in the period of dentistry graduation that the student sees itself faced with not only technical issues, but with health situations in a wholesome view, as in the case of wastes generated during clinical practice. The aggravations to professionals/students and staff, third parties, in the outdoors of service establishments, as well as in the environment, may occur if proper procedures in management of wastes produced in dentistry are not observed, given that the potential risk of these increases when they are handled inadequately or are not properly prepared and disposed of, particularly in situations which favor the penetration of risk agents in the human organism and/or harm to the environment. In the issue that involves these types of waste, knowledge is the essential tool for proper management actions, from minimizing production to correct disposal. In order to contribute how the knowledge can be inserted in the application of this theme in the dentistry professional formation, it's proposed a literature review, with descriptive, exploratory and bibliographic research of academic-scientific and normative materials related to the management of health waste in dentistry, associating it to the importance of approaching the issue in the Biosafety discipline, presenting its scope in promoting in human and environmental health, which constitutes as a relevant proposal in the context of public health.

Descriptors: Education in Dentistry. Biosafety. Waste of Health Services.

Correspondência para:

Carmen Angela Guimarães Leal

e-mail: cagleal@gmail.com

Rua Pina Leitão, 331

27600-000 Torres Homem, Valença, RJ. 